\title{
Development of the FPDA technique to study the atomization of emulsions
}

\author{
Marie Loustau',2, Virginel Bodoc², Geoffroy Illac², Jean-Paul Couput ${ }^{1}$, Pierre Gajan² \\ ${ }^{1}$ Metering and Allocation, Total SE, Pau, France \\ 2Département Multi-physique pour l'Energétique, Office National d'Etudes et de Recherches \\ Aérospatiales, Toulouse, France \\ ${ }^{*}$ Corresponding author email: marie.loustau10@gmail.com
}

\begin{abstract}
The aim of this study is to improve metering of liquid and gas quantities encountered along the gas production chain. When natural gas is extracted, the differences between reservoir and surface conditions are inducing the formation of an annular dispersed wet gas flow in the pipe. Thus, the presence of liquids (water and oil) is leading to an increase in the differential pressure measured in the Venturi flowmeter, inducing an "over-reading" that must be corrected for fiscal and allocation operations. One way to perform this correction consists in a flow modelling by taking into account the atomization process in the Venturi that significantly contributes to the over-reading. To characterize the dispersed phases, parameters such as droplets sizes, velocities and atomization rates need to be measured. For that, an innovative method named Fluorescence Phase Doppler Anemometry (FPDA) was developed. In this configuration, three detectors of the Dual Mode PDA are dedicated to droplets velocity and size measurement (through Mie scattering process), while the fourth one is used to identify the fluorescent droplets. Water was seeded with Rhodamine 6G (Fluorescent signal), while oil was not seeded (Mie Scattering signal). Thanks to this technique, velocities and diameters of two phases can be obtained. Experiments will be conducted in single-phase spray, and in dual-phase spray to validate the FPDA method.
\end{abstract}

\section{Keywords}

Liquid Atomization, Multiphase Flows, Fluorescence Phase Doppler Anemometry, Venturi, Wet Gas Flows

\section{Introduction}

In the oil \& gas industry, wet gas is referring to flows conditions where the Gas Volume Fraction (GVF) is higher than $95 \%$. In most cases, an annular/dispersed flow is observed which means that a mixture of gas and liquid droplets is flowing at the center of the pipe at high velocity and is surrounded by a thin water/oil liquid film flowing at low velocity along the pipe wall. In the film, the liquids can be encountered as stratified phases or as a water/oil emulsion depending on the operating conditions (watercut, flowrates, and pressure). Because they are robust, costeffective and simple devices, Venturi flowmeters are widely used to access the flowrates of each phase. In the oil \& gas industry, accurate measurement of wet gas and multiphase flows is of prior importance to improve operational efficiency and answer to the fiscal and allocation challenges.

For wet gas flows, the presence of liquids induces an increase in the differential pressure measured inside the Venturi, which is leading to an over-estimation of the gas mass flow rate that is called over-reading. For fiscal and allocation operations, this over-reading must be corrected. 
Different approaches are applied to estimate the over-reading and correct the flowrates of the different phases. The first is based on empirical correlations which were developed for flows at specific conditions which means that these correlations are valid for a specific pressure, temperature, and Venturi diameter [1][2]. As an example, De Leeuw correlation was the first correlation developed for Venturi meters, but this is only applicable for 4" Venturis [3]. Recently, an important effort has been made to improve the existing correlations for ISO:TR11583 for Venturi mounted vertically because most of the studies is about Venturi mounted horizontally [4]. The aim of their work was to provide a modification of the ISO correlation to reduce the over-reading for Venturis mounted vertically thanks to the results of an experimental campaign conducted for three different Venturi tubes. All these correlations enable to significantly reduce over-reading but this is valid for specific operating conditions. There is a need to improve the existing correlations to achieve a better accuracy in the determination of the flowrates.

A second approach is currently used in a numerical tool named "WegMove" under development by Total and ONERA [5][6]. It consists in the modelling of the physical phenomena occurring inside the Venturi such as the contribution of the liquid film atomization at the end of the convergent section that significantly contribute to the over-reading. This atomization induced by the slope breaking occurring inside the Venturi, depends on the operating conditions (watercut, flowrates, GVF). These conditions modify the atomization rate, the size of the droplets formed and consequently their interaction with the gas flow. The improvement of the three-phase flow modelling used in the code is mainly based on experimental correlations developed to determine the characteristics of the water/oil spray issued from this atomization process. This justify the development of measurements technique such as the Phase Doppler Anemometry (PDA) discriminating the contribution of each liquid to the atomization process.

The Phase Doppler Anemometry is an optical non-intrusive measurement technique based on the principle of light scattering that enables to characterize sprays with a high temporal resolution. Thanks to this technique, the diameters and velocities of a single-phase spray could be determined. To characterize two-phase flows and answer to the industry needs (oil and gas industry, automobile engines) the classic version of the PDA is not sufficient. The classic version of the PDA does not enable to discriminate the phases so that the characteristics of only one phase can be obtained simultaneously. In this frame Rottenkolber et al. [7] presented a fluorescent LDV-system (FLDV) that provides the velocity of the gas phase in the presence of the dispersed phase by adding fluorescent tracer particles to the gas flow. This approach was based on a spectral discrimination into Mie-scattered and fluorescent light of the recorded burst signals. Velocity evaluation for the disperse phase is done by analyzing the Doppler frequency in a standard Mie-scattering configuration.

Later on, Hehle et al. [8] have put an important effort to develop an upgraded configuration of the PDA to characterize two-phase flows. This technique named FPDA is combining the fluorescence with the classic PDA. The objective of the FPDA is to obtain the velocities and the diameters of the two phases at the same time and to discriminate the contribution of each liquid to the atomization process. Their experimental setup and their article are in line with the work done by Rottenkolber et al. [7]. Two nebulizers are used to produce fluorescent and nonfluorescent tracer particles to have a two-phase flow and to validate the experiments. A long pass filter has been mounted (at the backscatter of a standard fiber 2D transmitting probe or to the PDA receiving optics). This filter is not totally blocking the Mie scattering signals. In their work, they highlighted the difficulties to have a sufficient fluorescent signal because the intensity in fluorescence is proportional to $\mathrm{d}^{3}$ while for the Mie scattering the light intensity is proportional to $d^{2}$ which means that in certain cases Mie scattering particles could be detected 
while fluorescent signals are not sufficient to be detected. One solution to avoid these artefacts could be to place an additional PM (photomultiplier) in a direction where the Mie scattering signal is weak. An electronic trigger device was in charge of generating a TTL signal every time a fluorescent particle was passing through the measurement volume. Post-processing was developed and tested too. The work done by Hehle et al [8] is showing that FPDA is a powerful tool for future characterizations of two-phase flows.

The objective of the present work is to perform the phase discrimination as proposed by Rottenkolber et al. [7] and Hehle et al [8] and to adapt it to a PDA system. In this case a onedimensional velocity will be used.

The FPDA technique and the experimental campaign will be presented in detail in this paper. First, the tests will be conducted for a single-phase spray to validate the technique and the filtering algorithm developed, and then the technique will be applied to a dual-phase spray made of water and FV A50B.

\section{Material and Methods}

Fluids properties and FPDA technique principle

For these experiments, a $112 \mathrm{~mm}$ dual PDA from Dantec (BSA P800) was used. For the needs of the measurement technique validation, only the $532 \mathrm{~nm}$ wavelength laser beams were used. The PDA system is set up in first order refraction $\left(\sim 30^{\circ}\right)$ at an atmospheric test rig. Two different spray nozzles were mounted to generate two-liquid continuous flows with fluorescent and non-fluorescent drops.

To validate the technique, the experiments were carried out in a single-phase spray made of water seeded with Rhodamine 6G. In a second step a dual-phase spray of water seeded with Rhodamine 6G and Finavestan A50B oil (FV A50B) were tested. This oil has been selected to mimic the properties of standard condensates encountered in natural gas production facilities. The oil has been characterized in Total facilities (see Table 1). The Rhodamine 6G has been selected as a fluorescent dye because it is immiscible with FV A50B. For the Rhodamine $6 \mathrm{G}$, a concentration of $250 \mathrm{mg} / \mathrm{L}$ has been used in order to have enough signal intensity.

\begin{tabular}{c|cc}
\multicolumn{3}{c}{ Table a - Fluids properties at $20^{\circ} \mathrm{C}$ temperature } \\
\hline & Water & $\begin{array}{c}\text { Finavestan } \\
\text { A50B }\end{array}$ \\
\hline $\begin{array}{c}\text { Density } \\
\left(\mathrm{g} / \mathrm{cm}^{3}\right)\end{array}$ & 0.9983 & 0.8275 \\
\hline $\begin{array}{c}\text { Dynamic } \\
\text { Viscosity } \\
(\mathrm{mPa} \text { ) })\end{array}$ & 1.1523 & 11.6327 \\
\hline $\begin{array}{c}\text { Surface } \\
\text { Tension } \\
(\mathrm{mN} / \mathrm{m})\end{array}$ & 70.29 & 27.89 \\
\hline
\end{tabular}

The optical setup is schematically presented in Figure 1. The Mie and fluorescence bursts were simultaneously acquired with the PDA-system. Three of the four photodetectors are dedicated to droplets size and velocity measurements through a standard Mie-scattering process (PDA measurement), while the fourth one is devoted to identify the fluorescent droplets (see Figure 1). In this case, the fourth photodetector mounted to the PDA receiving 
optics is equipped with a long pass interferential filter $(\lambda>532 \mathrm{~nm})$ selected according to the reemission properties of the fluorescent dye (Rhodamine 6G) (see Figure 2). In the BSA software, the fluorescent burst analysis does not enable the phase discrimination immediately, but the envelope of the fluorescent bursts is used to generate a TTL signal every time a fluorescent droplet is detected. The arrival time, size, velocity signals determined from the Mie-scattering process and TTL signals deduced from the fluorescent process are simultaneously acquires by the BSA processor. An appropriate post-processing algorithm for phase discrimination is then applied offline. For this experimental campaign, the gain and the amplification tension for the photodetector designated for the fluorescence recording was set to $18 \mathrm{~dB}$ and $1800 \mathrm{~V}$. These parameters were chosen to get a strength enough fluorescence burst.

The arrival time (AT) of the droplet in the measurement volume through a standard Mie scattering process is compared to the arrival time of the fluorescent droplets (Sync_AT). If Sync_AT belongs to the interval [AT; AT+delta_t] (with delta_t an operator defined time lag depending on the operating conditions) then it can be considered as corresponding to a fluorescent droplet.

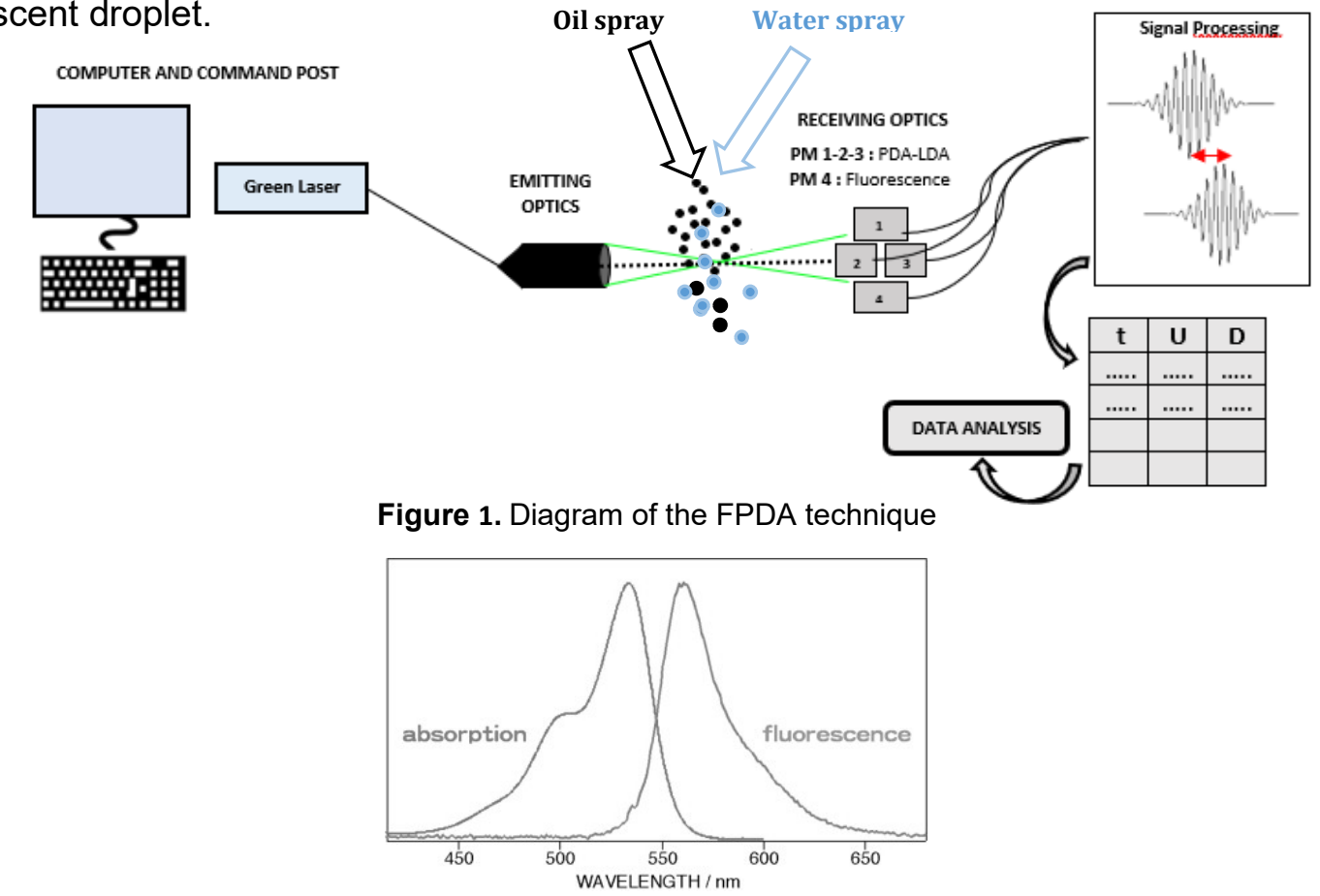

Figure 2. Absorption and emission spectra of Rhodamine $6 \mathrm{G}$ at a laser wavelength of 532nm [9]

\section{Experimental campaign}

The measurements where done both for a single-phase spray made of water seeded with Rhodamine 6G and for a dual-phase spray (made of water and FV A50B). The probe volume was placed downstream the injection nozzles at a sufficient distance in order to guarantee the presence of spherical droplets (see Figure 3). Following this figure, the origin of the basis is situated at the exit of the water spray, and the probe volume is situated in the plan containing the injection axis of the two injectors (which means $x=0 \mathrm{~mm}$ ) at $y=-20 \mathrm{~mm}$ and $z=70 \mathrm{~mm}$.

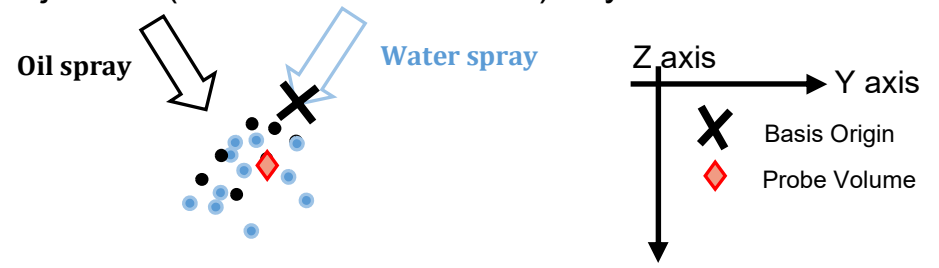

Figure 3. FPDA setup and measurement volume localization 


\section{Post-processing routine}

When a droplet is crossing the probe volume, it scatters light that is then received by the different photomultipliers (PMs), located at specific positions $\left(30^{\circ}\right.$ in this case), to collect the maximum light intensity. An electrical signal is generated, this is called a burst. The properties of the bursts enable to have the properties of the droplets : the frequency of the burst and the distance between the fringes enable to compute the speed of the droplet, while the phase difference between two bursts is directly linked to the diameter of the droplet. Thus, the PDA software is generating a table with the characteristics of the droplets seen by the three photodetectors. The Arrival Time is corresponding to the arrival time of the droplet in a standard Mie scattering process in the measurement volume.

While when a fluorescent drop crosses the probe volume the fluorescence burst generates a TTL signal (see Figure 4). During operation, it was observed that the generation of the TTL signals may be an important source of "ghost" fluorescent drops which means that for a noisy fluorescence burst (due to an important gain and/or tension on the PM) several TTL gates are generated by the PDA hardware. This effect is conducting to a higher number of fluorescent drops than the amount expected. Because this effect cannot be avoided by any electronic solutions, an alternative solution was adopted. It consists in a filtering algorithm developed under MatLab software to compare the arrival time AT as "seen" by the non-filtered PMs with Sync AT (the arrival time of the fluorescent particle) as "seen" by the filtered PM. This algorithm helps to delete the multiple TTL signals generated for one droplet to keep only one TTL signal for the fluorescent particles.

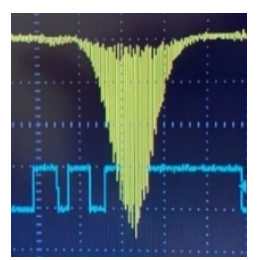

Figure 4. Yellow line: fluorescence burst - Blue line : TTL signals corresponding to the fluorescence burst

Once this filtering is done, the algorithm creates four tables: speed of the fluorescent droplets, speed of the Mie scattering droplets, diameter of the fluorescent droplets, and diameter of the Mie scattering droplets. This enables to draw the corresponding distributions: droplets population as a function of diameters, and droplets population as a function of the speed. To understand the filtering algorithm main objectives, see Figure 5.

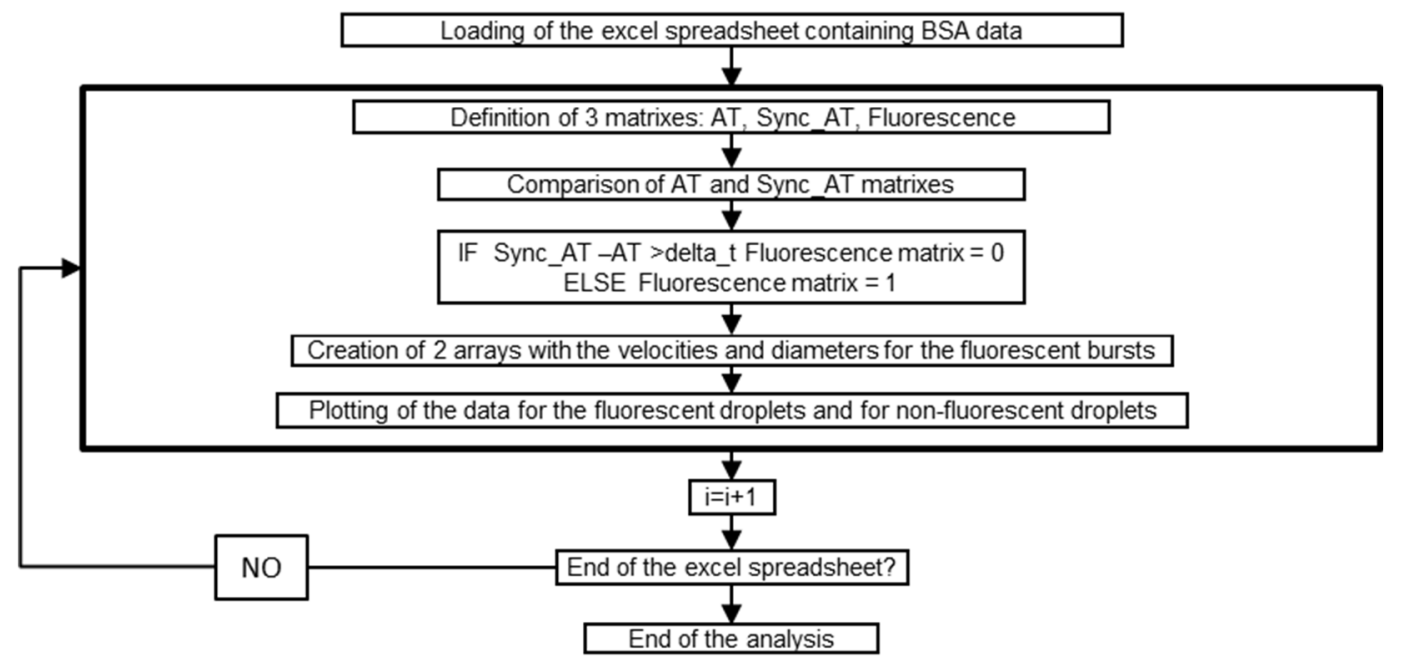

Figure 5. Flowchart of the filtering algorithm 


\section{Results and Discussion}

Experiments with non-seeded water filtering

In a first step the experimental setup (optical setup and post-processing algorithm) was validated on a non-seeded water spray. In this case the water was not seeded with dye, and no optical filter was placed on the dedicated PM. As in a classical way, the fourth photodetector is detecting water droplets through a Mie scattering process. The bursts of the first three detectors are processed to compute drops velocities and diameters (phase discrimination) and the arrival time. For the fourth burst, the parameter of interest is only the arrival time. On the two series of arrival times the filtering algorithm was applied. The results of the experiments are presented in Figure 6.
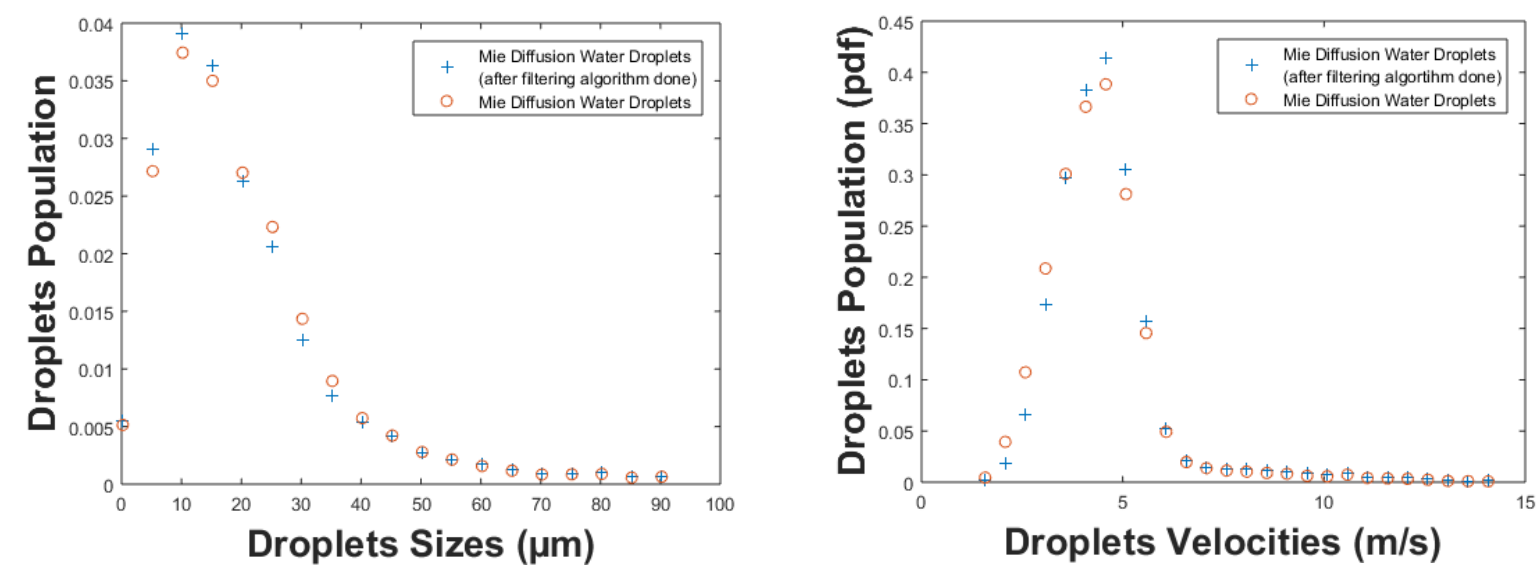

Figure 6. Left: Droplet population as a function of droplets sizes. Right: Droplet population as a function of droplets velocities. Experiments done in Mie Scattering exclusively with a pure water spray.

Figure 6 compares the droplets sizes and velocities histograms obtained for the same water drops before and after application of the filtering algorithm. As expected for each graphs the two histograms are identical which indicates that the filtering algorithm does not alter the information on the drops characteristics as size and velocity. Thus, the filtering algorithm is validated for a Mie burst.

The next step in the validation of the FPDA technique was the use of seeded water as test liquid. In this case, the water spray was seeded with Rhodamine 6G and the long-pass filter was placed on the fourth photodetector. The aim of this experiment is to validate that the application of the filtering method on the fluorescent burst does not alter the size and velocity distributions measurement. The results are presented in Figure 7 that compares the droplets size and velocity histograms obtained before (PDA data), and after applying the filtering algorithm (FPDA data). Only $1.6 \%$ of the droplet population is not detected by the fourth photodetector. This concerns mainly small droplets whose fluorescent reemission (proportional to $\mathrm{d}^{3}$ ) is weak. In consequence only a small differences appear on the mean values calculated from these two droplet samples $\left(0,04 \%\right.$.for $d_{32}$ and the $0,98 \%$ for the $\left.d_{10}\right)$. In consequence, this artefact will result in a small measurement bias on the determination of averaged droplet size and velocity values. 

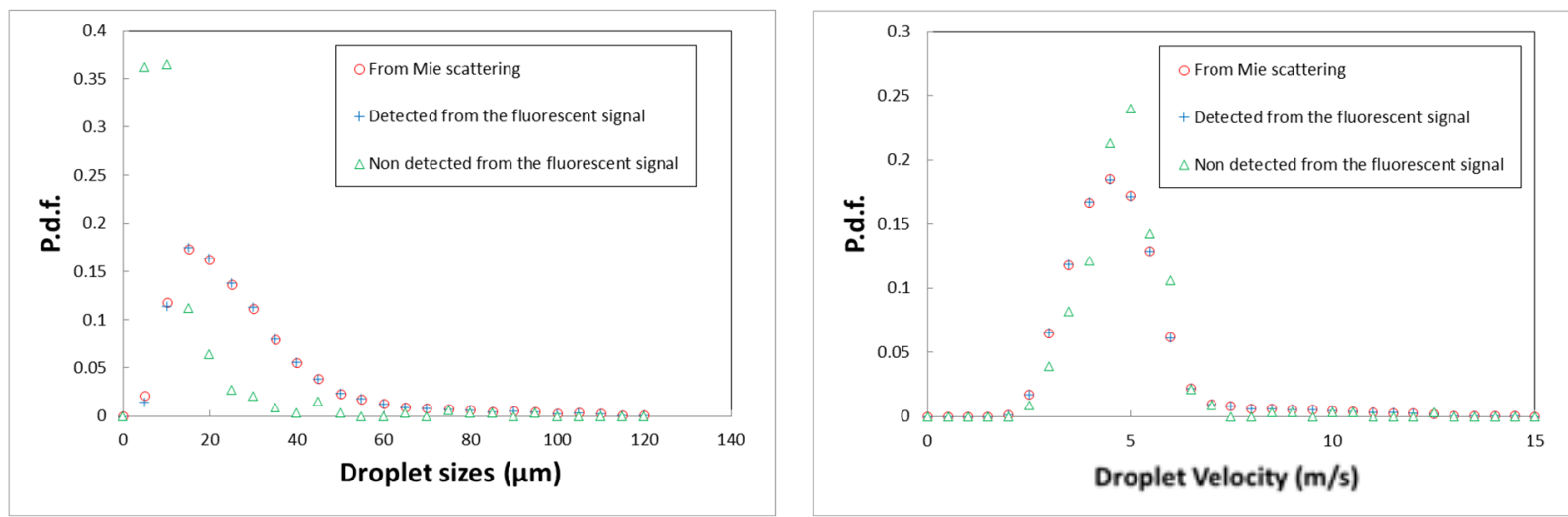

Figure 7. Left : Droplet population as a function of droplets diameters. Right : Droplet population as a function of droplets velocities. Comparison of the distributions for the fluorescent droplets and for the droplets through a standard Mie scattering process.

The FPDA method was applied for a real two-phase flow. The two sprays (water seeded with Rhodamine 6G and FV A50B) are injected simultaneously in order to compare their characteristics. The results of the experiments are presented in Figure 8. Three series of data corresponding to the global spray and to each phase (water and oil) are compared on the same graph. The characteristics (size and velocity) of the global spray are those provided directly by the PDA with no filtering. As for the individual phases the characteristics were obtained after application of the filtering approach (FPDA).
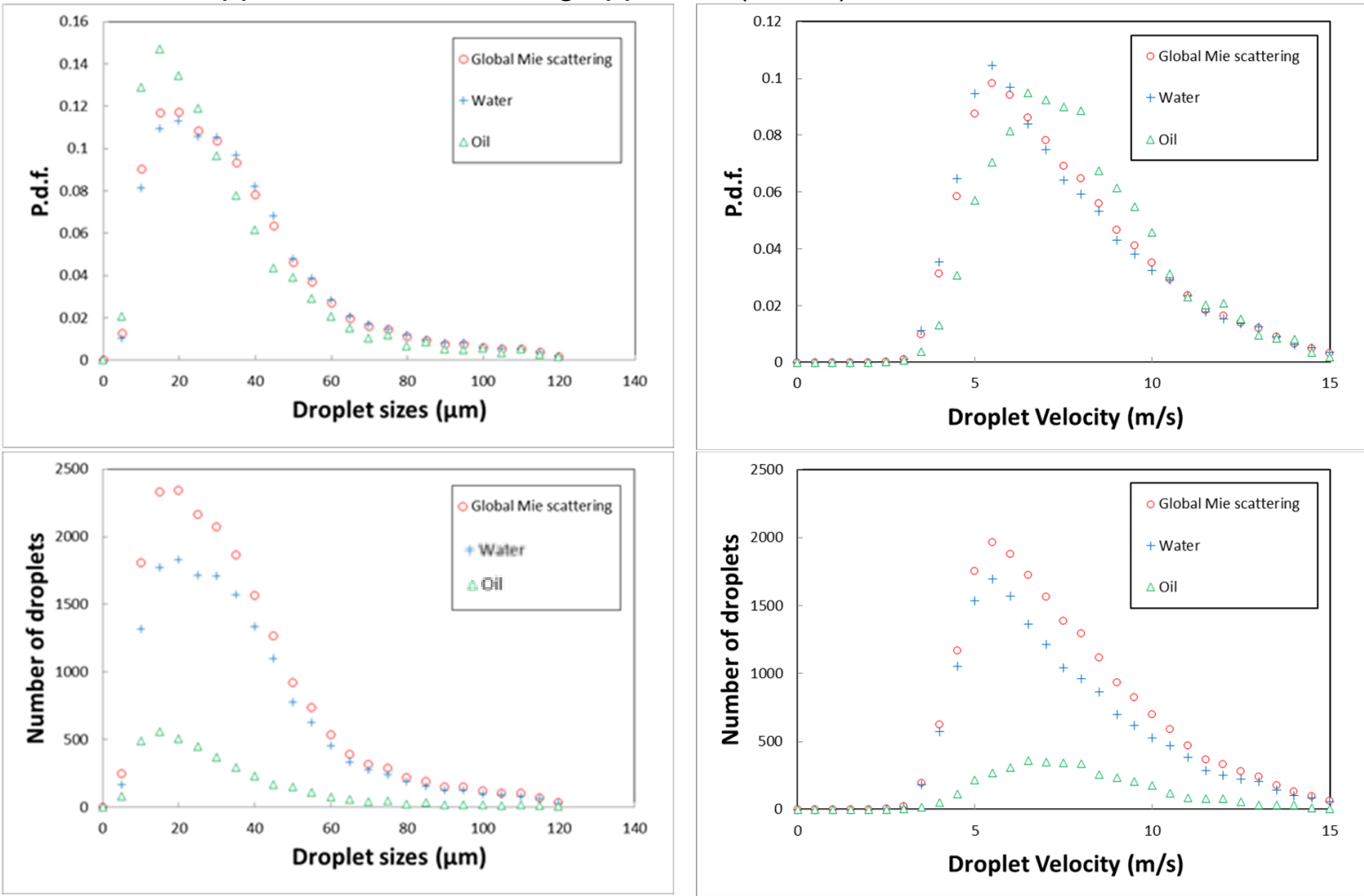

Figure 8. Left: Droplet population (PDF) as a function of droplets sizes. Right: Droplet population (pdf) as a function of droplets velocities. Comparison of the distributions for the fluorescent droplets (water) and for the nonfluorescent droplets (oil) and for global Mie scattering (water and oil).

Some differences appear between the water and FV A50B distributions which mainly concern the small droplets. Moreover, the lower graphs representing the distributions in terms of number of droplets, show that a lower quantity of oil drops (about 19.0\%) is detected on the treated sample. On the velocity distributions, it seems that the oil droplets move faster in the spray at this probe location. The mean diameter $d_{32}$ for oil droplets is equal to $59,7 \mu \mathrm{m}$ and 
$61,7 \mu \mathrm{m}$ for water droplets. For the velocities, the average velocity for oil droplets is equal to $7,64 \mathrm{~m} / \mathrm{s}$, while for water droplets it is $7,13 \mathrm{~m} / \mathrm{s}$.

\section{Conclusions}

As a conclusion, we can highlight the main advantage of the FPDA technique that is to access the characteristics (diameter and velocity) of the two-phases (in our case water and FV A50B) at the same time, while for a standard PDA configuration the characteristics of only one phase could be determined.

The innovative FPDA technique is working in the two cases (water spray and bi-spray) and the filtering algorithm developed on MatLab is correctly functioning. However, we can underline that there is a small tendency to underestimate the fluorescent small droplets, but this influence is week. The application of this method on a bi-fluid spray allows to isolate the droplets from each of the considered fluids and to calculate their respective distribution and to determine their respective mean droplet size and velocity.

The next steps will be dedicated to the implementation of the technique in the multiphase test flow loop at Onera lab, to characterize the atomization process occurring at the convergent section of the Venturi to improve the current existing models in WegMove code.

\section{Acknowledgments}

The authors thank TOTAL company for their financial support and ONERA laboratory for the facilities.

\section{Nomenclature}

GVF Gas Volume Fraction

PDA Phase Doppler Anemometry

PM Photomultiplier

PDF Probability Density Function

FPDA Fluorescence Phase Doppler Anemometry

\section{References}

[1] J. W. Murdock, "Two-Phase Flow Measurement With Orifices," J. Basic Eng., vol. 84, no. 4, pp. 419-432, Dec. 1962.

[2] D. Chisholm, "Flow of Incompressible Two-Phase Mixtures through Sharp-Edged Orifices," J. Mech. Eng. Sci., vol. 9, no. 1, pp. 72-78, Feb. 1967.

[3] R. de Leeuw, "Liquid Correction of Venturi Meter Readings in Wet Gas Flow," in NSFMW, 1997, p. 335.

[4] E.M. Graham, M. Reader-Harris, G. Chinello, K. Harkins, N. Bowman, L. Wales, Vertically installed Venturi tubes for wet-gas flow measurement: Possible improvements to ISO/TR11583 to extend its range of applicability, J. Flow Meas. and Inst., vol. $74,2020,101757$,ISSN 0955-5986

[5] Salque, Geraldine \& Couput, J.-P \& Gajan, Pierre \& Strzelecki, A. \& Fabre, J.-L. (2007). New correction method for wet gas flow metering based on two phase flow modelling: Validation on industrial air/oil/water tests at low and high pressure. Energy Institute - 26th NSFMW 2008. 292-309.

[6] Couput, J.-P \& Tanguy, B \& Gajan, Pierre (2014). Venturi flow meters behaviour in high pressure 3 phases wet gas conditions. 32 nd NSFMW 2014.

[7] Rottenkolber, G. \& Meier, Robert \& Schäfer, O. \& Wachter, S. \& Dullenkopf, Klaus \& Wittig, S.. (2002). Combined PDA and LDV measurements: Phase discrimination inside a spray using fluorescent seeding particles.

[8] M. Hehle, M. Ochs, O. Schäfer, K. Dullenkopf and H.-J. Bauer, Development of an enhanced pda-system for simultaneous measurement of both phases in two-phase flows by Lehrstuhl und Institut für Thermische Strömungsmaschinen Universität.

[9] M. Sakai, T. Ohmori, M. Fujii, Chapter 12 - Two-color picosecond time-resolved infrared super-resolution microscopy, Handai Nanophotonics, Elsevier,Volume 3, 2007. 